This is an Accepted Manuscript of an article published by Taylor \& Francis Group in Journal of Hydraulic Research on 22/07/2020, available online:

https://www.tandfonline.com/doi/full/10.1080/00221686.2019.1647885.

\title{
Transverse surface waves in steady uniform and non- uniform flows through an array of emergent and slightly submerged square cylinders
}

\author{
MERIEM CHETIBI ${ }^{1}$, SEBASTIEN PROUST, SAADIA BENMAMAR.
}

\begin{abstract}
Steady uniform and non-uniform flows through an array of emergent and slightly submerged square cylinders are experimentally investigated with a specific focus on transverse seiche waves induced by vortex shedding. The study is first and foremost aimed at assessing the effect of streamwise flow non-uniformity on seiche waves. Its secondary purpose is to investigate the change in seiche magnitude, when initially emerged cylinders become slightly submerged. Thirdly and lastly, the effect of seiche waves on mean velocities and velocity fluctuations is quantified. The lock-in process between waves and vortex shedding is unaltered by flow non-uniformity and by a change from cylinder emergence to submergence. For non-uniform flows, this results in the co-existence of two differently oscillating transverse waves close to each other. Relative wave amplitude is found to be mainly influenced by relative submergence in the case of submerged cylinders, and by Froude number and oscillation mode in the case of emergent cylinders. Finally, seiche waves modify the streamwise mean velocity, when cylinders are emergent.
\end{abstract}

keywords : Non-uniform flow; open-channel flow; relative submergence; seiche; surface wave; vortex shedding.

\footnotetext{
${ }^{1}$ Email: meriem.chetibi@g.enp.edu.dz
} 
This is an Accepted Manuscript of an article published by Taylor \& Francis Group in Journal of Hydraulic Research on 22/07/2020, available online: https://www.tandfonline.com/doi/full/10.1080/00221686.2019.1647885.

\section{Introduction}

The periodic free surface oscillations observable in laterally confined steady open-channel flows are also termed the 'seiche phenomenon'. Ilich (2006) states that a seiche is the oscillating response of an enclosed body of water, which acts to return the system to equilibrium. Rabinovich (2009) defines a seiche as 'long-period standing oscillations in an enclosed basin or in a locally isolated part of a basin'. Seiches have been exhaustively studied in not only lakes and harbors (e.g. Chen, Lee, Wu, \& Lee, 2013; G. Wang, Zheng, Liang, \& Zheng, 2014), but also in laboratory open-channel flumes in the case of streamwise uniform flows through arrays of emerged circular cylinders (e.g. Dupuis, Proust, Berni, \& Paquier, 2016; Ghomeshi, Mortazavi-Dortazavi, \& Falconer, 2007; Jafari, Ghomeshi, Bina, \& Kashefipour, 2010; Viero, Pradella, \& Defina, 2016; Zhao, Cheng, \& Huang, 2014; Zima \& Ackermann, 2002) or submerged circular cylinders (Jafari et al., 2010; Sarkar, 2012). The seiche phenomenon is a physical process of prime importance in laterally confined open-channel flows since the seiche amplitude $A$ can reach $40 \%$ of the timeaveraged flow depth $D$ (Jafari et al., 2010).

In the case of flows through arrays of cylinders, a seiche is a resonance phenomenon between vortex shedding and natural transverse surface waves in the channel. Resonance occurs when the vortex shedding frequency is close to the natural transverse wave frequency, $f_{t w}$, which is dependent on channel width $B$, flow depth $D$ and oscillation mode $n$ (e.g. Dean \& Dalrymple, 1991). Vortex shedding frequency tends towards $f_{t w}$ and the lock-in process occurs, when both frequencies are the same.

This paper reports a laboratory investigation of transverse waves developing in steady uniform and non-uniform open-channel flows through arrays of square cylinders installed on a rough bed. The square cylinders represented house models extending across urbanized floodplains. This study is part of a research project on predicting floodplain flows during extreme flooding events (Proust et al., 2016, October). To the authors' knowledge, the seiche phenomenon caused by square cylinders has not been studied before. The originality of the study does not lie in the cylinders' cross-sectional shape, but in the following three key issues.

The first embodies the question of what effect does streamwise flow nonuniformity have on seiche waves and the associated lock-in process. This question is legitimate because streamwise non-uniform flows are very common in flooded natural or urbanized floodplains (e.g. Dupuis et al. (2016) for 
This is an Accepted Manuscript of an article published by Taylor \& Francis Group in Journal of Hydraulic Research on 22/07/2020, available online: https://www.tandfonline.com/doi/full/10.1080/00221686.2019.1647885.

non-uniform flows through floodplain woodland). The second key issue involves what happens to seiche waves and the associated lock-in process, when initially emerged square cylinders become slightly submerged. When dealing with real floodplains, submergence of floodplain roughness elements can vary with the return period $T$ of a flooding event (e.g. emerged house for a given return period $T$ and slightly submerged house for a longer return period). The third and final key issue involves what effect do transverse waves have on time-averaged velocities and velocity fluctuations over the water column. The object of this paper is to attempt to consider the three issues based on laboratory experiments.

The experiments were started under streamwise uniform flow conditions, i.e. with a constant relative cylinder submergence $D / h$ in the longitudinal direction (where $h$ is the height of a square cylinder). Two flow cases were studied with $D / h=0.98$ and 1.2. The case of slight submergence of roughness elements is challenging since it features the complex vertical structure represented by the turbulent boundary layer above and below the top of the roughness elements, as observed by Florens, Eiff, and Moulin (2013) for $D / h=3.0$ to 6.7 , by Rouzes, Moulin, Florens, and Eiff (2018) for $D / h=1.5$ to 3.0 and by Chagot, Moulin, and Eiff (2017, June) for $D / h=1.1$ to 4.1 . It should be noted that these authors focused on flow structure without quantifying surface waves.

We then investigated streamwise non-uniform flows (essentially accelerated flows) with the same flow rates as in the uniform scenarios, but with a different downstream weir height. The cylinders were essentially emergent in two flow cases, but a longitudinal transition from slightly submerged to emergent cylinders occurred in two other flow cases.

The paper outlines the experiments (flume, flow conditions, flow depth and velocity measurements) before providing the theoretical background regarding seiche wave parameters and square cylinders. Section 4 includes the results pertinent to the above three key issues. A number of conclusions are ultimately provided. 
This is an Accepted Manuscript of an article published by Taylor \& Francis Group in Journal of Hydraulic Research on 22/07/2020, available online:

https://www.tandfonline.com/doi/full/10.1080/00221686.2019.1647885.

\section{Experiments}

\subsection{Experimental facility}

The experiments were conducted in an $18 \mathrm{~m}$ long $\times 1 \mathrm{~m}$ wide open-channel flume (Fig. 1a) in the Hydraulics and Hydromorphology Laboratory at Irstea Lyon-Villeurbanne, France. The longitudinal bed slope $S_{0}$ was $1.05 \times 10^{-3}$. The rectangular cross section was bounded by glass sidewalls and a bottom covered by dense synthetic grass (rigid blades of height $\xi=5 \mathrm{~mm}$, see Fig. 1b), which modeled a dense meadow. Square cylinders representing house models were then installed in an in-line arrangement on the synthetic grass. Each cylinder had a square cross section (side length $l=64 \mathrm{~mm}$, see Fig. 1c) and its height $h$ was $59.2 \mathrm{~mm}$ (measured from the top of grass blades, see Fig. 1b). The distance $L$ between the centers of two adjacent cylinders was $143 \mathrm{~mm}$ (Fig. 1c). The cylinder planar density $\left(\lambda=l^{2} / L^{2}\right)$ was 0.20 and the number of cylinders per square meter was $49(7 \times 7)$. The cylinder frontal density was $\lambda_{\mathrm{f}}=(D l) / L^{2}$ for the emergent cylinders and $\lambda_{\mathrm{f}}=(h l) / L^{2}$ for the submerged cylinders $\left(\lambda_{\mathrm{f}}\right.$-values are given in Table 1$)$.

The values of $\lambda$, dimensions $l$ and $L$, and the in-line arrangement of the array were subject to several constraints. The $\lambda$-value was established for several experimental setups used in the same research project (see section 1). Dimensions $l$ and $L$, and the in-line arrangement were chosen such that Acoustic Doppler Velocimeter (ADV) measurements could be readily taken between two adjacent rows of cylinders. Finally, the highest value of $l$ was chosen because we wanted the width of each house model to be large with respect to other characteristic lengths, e.g. the model synthetic grass height $\xi$ and tree model diameter (see Dupuis et al. (2016)).

A Cartesian coordinate system was set up. The $x, y$, and $z$ axes represented the longitudinal (parallel to flume bottom), crosswise and vertical (normal to flume bottom) directions respectively. The origin was defined as: $x=0$ located $75 \mathrm{~cm}$ downstream of the inlet tank outlet, cylinder centers of the first lateral row located at $x / B=0.2, y=0$ located on the right-hand sidewall of the channel and $z=0$ located at the tips of the synthetic grass blades. 
This is an Accepted Manuscript of an article published by Taylor \& Francis Group in Journal of Hydraulic Research on 22/07/2020, available online: https://www.tandfonline.com/doi/full/10.1080/00221686.2019.1647885.

(a)

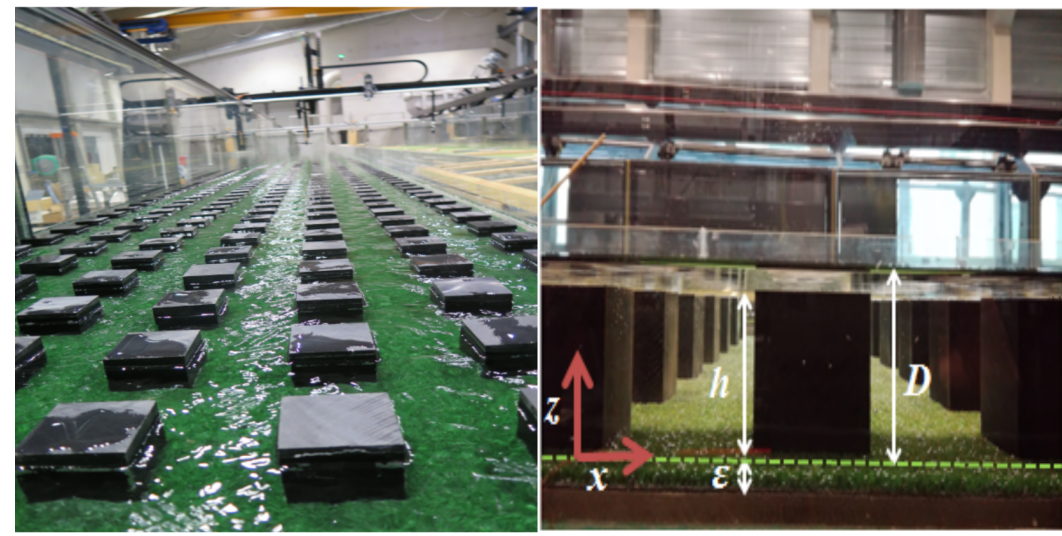

(c)

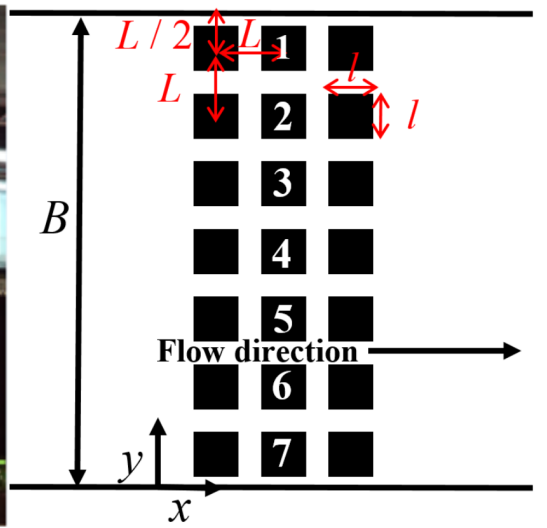

Figure 1: View of open-channel flume looking upstream: (a) uniform flow with relative submergence $D / h=0.98$; (b) side view of uniform flow with $D / h=1.20$; and (c) sketched top view of the in-line distribution of square cylinders.

Table 1: Test case flow conditions: $Q$ is discharge, $D$ is time-averaged water depth, $D / h$ is relative submergence, $U_{\lambda}$ is average pore velocity, $\lambda_{\mathrm{f}}$ is frontal density of square cylinders, $\mathrm{F}_{\mathrm{D}}=U_{\lambda} / \sqrt{g D}$ is Froude number, $\mathrm{R}_{\mathrm{l}}=U_{\lambda} l / \nu$ is Reynolds number and $W$ is the downstream weir height. Variations between $x / B=0.27$ and $x / B=16.58$ for the accelerated flows

\begin{tabular}{|c|c|c|c|c|c|c|c|c|}
\hline Test cases & $Q\left(\mathrm{~L} \mathrm{~s}^{-1}\right)$ & $D(\mathrm{~mm})$ & $D / h$ & $U_{\lambda}\left(\mathrm{m} \mathrm{s}^{-1}\right)$ & $\lambda_{\mathrm{f}}$ & $\mathrm{F}_{\mathrm{D}}$ & $\mathrm{R}_{\mathrm{l}}$ & $W(\mathrm{~mm})$ \\
\hline UQ1W40 & 3.5 & 58 & 0.98 & 0.08 & 0.18 & 0.10 & 5120 & 40 \\
AQ1W20 & 3.5 & $51-38$ & $0.86-0.64$ & $0.09-0.12$ & $0.16-0.12$ & $0.12-0.19$ & $5760-7680$ & 20 \\
AQ1W10 & 3.5 & $51-28$ & $0.86-0.47$ & $0.09-0.16$ & $0.16-0.09$ & $0.12-0.30$ & $5760-10240$ & 10 \\
UQ2W47 & 5.7 & 71 & 1.20 & 0.10 & 0.19 & 0.12 & 6400 & 47 \\
AQ2W20 & 5.7 & $68-46$ & $1.13-0.78$ & $0.10-0.15$ & $0.19-0.14$ & $0.13-0.23$ & $6400-9600$ & 20 \\
AQ2W10 & 5.7 & $68-36$ & $1.13-0.61$ & $0.10-0.20$ & $0.19-0.11$ & $0.13-0.33$ & $6400-12800$ & 10 \\
\hline
\end{tabular}


This is an Accepted Manuscript of an article published by Taylor \& Francis Group in Journal of Hydraulic Research on 22/07/2020, available online:

https://www.tandfonline.com/doi/full/10.1080/00221686.2019.1647885.

\subsection{Flow conditions}

Flow conditions are reported in Table 1. Experiments were started under streamwise uniform flow conditions (constant relative submergence $D / h$ along the $x$-axis). The two cases investigated featured relative submergences $D / h=0.98$ and 1.20 , corresponding to flow rates $Q_{1}=3.5 \mathrm{~L} \mathrm{~s}^{-1}$ and $Q_{2}=5.7 \mathrm{~L} \mathrm{~s}^{-1}$ respectively. The heights of the downstream weir, $W$, which was set to give a water surface profile parallel to the flume bottom, were 40 $\mathrm{mm}$ and $47 \mathrm{~mm}$ for the above two flow rates respectively. The uniform cases are denoted UQ1W40 and UQ2W47 respectively ('U' referring to uniform flow).

Four non-uniform flows were subsequently investigated, keeping the same flow rates as for the uniform scenarios, but changing the heights of the downstream weir ( $W=10 \mathrm{~mm}$ and $20 \mathrm{~mm}$ for both flow rates $Q_{1}$ and $Q_{2}$ ). These non-uniform cases are denoted AQ1W20, AQ1W10, AQ2W20, and AQ2W10 ('A' referring to accelerated flow). The longitudinal profiles of $D / h$ are shown in Fig. 2a (see also the $D / h$-values in Table 1 ). The cylinders are essentially emerged for the accelerated cases with the flow rate $Q_{1}$. The cases with the flow rate $Q_{2}$ exhibit a longitudinal transition from slight cylinder submergence to emergence. It should be noted that the UQ1W40 case has the same $D / h$-value as the AQ2W20 and AQ2W10 cases at downstream position $x / B=13.43$ and 12.14 respectively (Fig. 2a).

\subsection{Flow depth measurements}

Flow depth was measured using an ultrasonic sensor (BAUMER UNDK 2016903/S35A). According to the manufacturer, sensor resolution is better than $0.3 \mathrm{~mm}$ and its reproducibility is better than $0.5 \mathrm{~mm}$. The maximum acquisition rate $(50 \mathrm{~Hz})$ was chosen to capture small wavelength waves. At each measuring point, the acquisition duration was chosen to achieve convergence in time of both the time-averaged flow depth, $D$, and the standard deviation of water depth fluctuations, $\sigma$. This duration varied between $60 \mathrm{~s}$ and $140 \mathrm{~s}$ depending on flow conditions (fast stream vs. wake zone, uniform vs. non-uniform flow, submergence value). It should be noted that the seiche can develop for $1000 \mathrm{~s}$ to $9000 \mathrm{~s}$ before reaching equilibrium (5 hours were required for flows through circular cylinders in the same flume, see Dupuis et al., 2016).

The transverse seiche wave was quantified at a given $x / B$-position by 
This is an Accepted Manuscript of an article published by Taylor \& Francis Group in Journal of Hydraulic Research on 22/07/2020, available online: https://www.tandfonline.com/doi/full/10.1080/00221686.2019.1647885.

its amplitude $A$ and by the maximum value $\sigma_{\max }$ of the standard deviation $\sigma$ observed across the channel width. The raw instantaneous flow depth $D(t)$ data were filtered; only data in the $D \pm 2 \sigma$ range were taken into account. New values of $\sigma$ and $\sigma_{\max }$ are then computed from the filtered data. It should be noted that wave amplitude $A$, which is the maximum oscillation observed at anti-nodes, is approximately equal to $4 \sigma_{\max }$ (Fig. $2 \mathrm{~b}$ ) since the free surface elevation oscillates between $-2 \sigma_{\max }$ and $+2 \sigma_{\max }$ at the anti-nodes.

The longitudinal profiles of flow depth were measured at three lateral positions: $y / B=0.29$ (between the second and third cylinders in a transverse row, see array numbering in Fig. 1c), $y / B=0.57$ (between the fourth and fifth cylinders) and $y / B=0.89$ (between the sixth and seventh cylinders). Measuring points were always located between two lateral rows of cylinders along the $x$-axis and the interval between two points varied from $L$ to $4 L$. Finally, the time-related convergence tests on $D$ and $\sigma$ were performed at downstream positions $x / B=8.57$ and $x / B=12.14$, and at $y / B=0.5$ (wake area behind a cylinder) and at $y / B=0.57$ (high velocity stream between two cylinders).

(a)

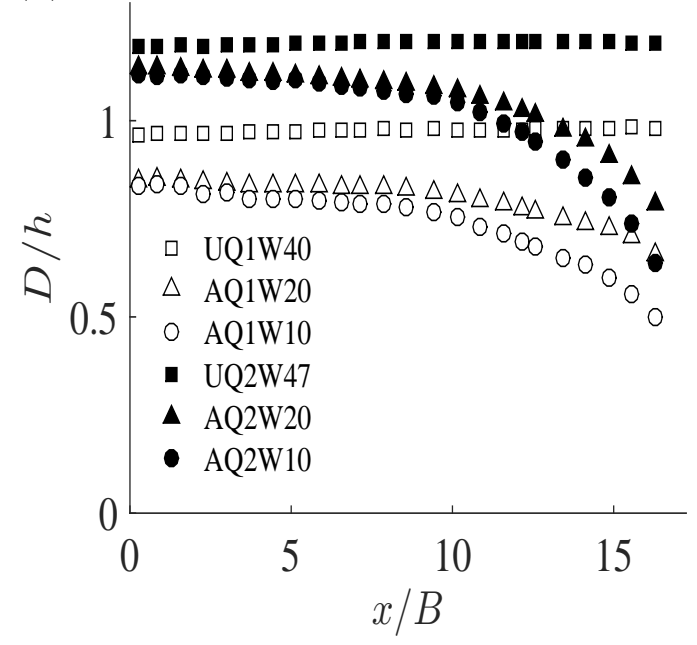

(b)

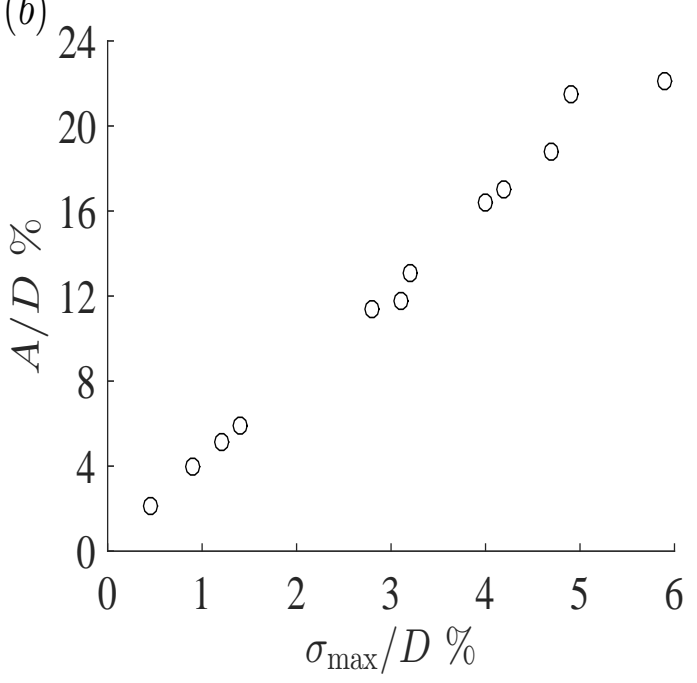

Figure 2: (a) Relative submergence $D / h$ with respect to downstream position $x / B$ for uniform flow cases (UQ1W40 and UQ2W47) and accelerated flow cases (AQ1W20, AQ1W10, AQ2W20, AQ2W10), average values at 3 $y / B$-positions (see section 2.3); (b) Linear relationship between $A / D$ and $\sigma_{\max } / D$. 
This is an Accepted Manuscript of an article published by Taylor \& Francis Group in Journal of Hydraulic Research on 22/07/2020, available online:

https://www.tandfonline.com/doi/full/10.1080/00221686.2019.1647885.

\subsection{Velocity measurements}

Velocities were measured using an Acoustic Doppler Velocimeter (ADV) with a side viewing probe (NORTEK VECTRINO +). According to the manufacturer, the uncertainty in velocity is $0.5 \%$ of the measured value $\left( \pm 1 \mathrm{~mm} \mathrm{~s}^{-1}\right)$. The sampling volume of an ADV can be approximated as a circular cylinder $(6 \mathrm{~mm}$ in diameter and $7 \mathrm{~mm}$ long in the transverse direction); the cylinder center is located $5 \mathrm{~cm}$ from the probe in the lateral direction. The acquisition rate was $100 \mathrm{~Hz}$ at each measuring point; this enabled velocity fluctuations with frequencies less than $50 \mathrm{~Hz}$ to be captured. The $200 \mathrm{~s}$ acquisition duration allowed for time-related convergence of both time-averaged velocity and second-order turbulence quantities in the free stream and in the wake area. The ADV data were post-processed using the WinADV software and de-spiked using the Goring and Nikora (2002) phase-space thresholding method. The flow was seeded with polyamide particles (Vestosint) with a median diameter of $40 \mu \mathrm{m}$ to increase the signal-to-noise ratio. Measurements were taken from $y / B=0.29$ to $y / B=0.57$ at spatial intervals of 16 $\mathrm{mm}$ and $32 \mathrm{~mm}$ along $\mathrm{y}$-axis in the free stream and wake zone respectively and at intervals of $3 \mathrm{~mm}$ over the flow depth.

\section{Theoretical background}

\subsection{Wavelength and frequency of transverse standing waves}

Transverse standing waves are characterized by nodes and anti-nodes, at which the free surface oscillation amplitude is minimum and maximum and the transverse velocity fluctuation is maximum and minimum respectively. Water motion is entirely horizontal at nodes and entirely vertical at antinodes (Rabinovich, 2009). It should be noted that two anti-nodes are always observed at the channel sidewalls owing to the zero-flow condition. The transverse wavelength is defined (e.g. Dean \& Dalrymple, 1991) by:

$$
\gamma=2 B / n
$$

where $n$ is the oscillation mode, i.e. the number of nodes.

Based on the small-amplitude water wave theory, wavelength can also be 
This is an Accepted Manuscript of an article published by Taylor \& Francis Group in Journal of Hydraulic Research on 22/07/2020, available online: https://www.tandfonline.com/doi/full/10.1080/00221686.2019.1647885.

related to frequency (Dean \& Dalrymple, 1991) as follows:

$$
\gamma=\frac{g}{2 \pi} \frac{1}{\left(f_{t w}\right)^{2}} \tanh \left(\frac{2 \pi D}{\gamma}\right)
$$

Combining Eq. (1) and Eq. (2) gives the frequency $f_{t w}$ as a function of oscillation mode. This relation has been tested against experimental data, e.g., by Zima and Ackermann (2002) for circular cylinders. It was recently adjusted by Zhao et al. (2014) to account for porosity:

$$
f_{t w}=\sqrt{\frac{\frac{g n}{4 \pi B} \tanh \frac{n \pi D}{B}}{S}}
$$

where $S=1+C_{A}(\lambda /(1-\lambda))$ is an inertial coefficient of the porous medium, and $C_{A}$ is a shape parameter related to added mass. According to Dean and Dalrymple (1991), $C_{A}=1$ for a square cylinder. In our experiments, $S$ equals to 1.25 as $\lambda=0.2$.

It should be noted that, when $S=1$ and $\gamma / D>20$, Eq. (3) gives the canonical Merian's frequency $f_{t w}=n \sqrt{g D} /(2 B)$, also called the frequency of long waves in shallow water (Dean \& Dalrymple, 1991).

\subsection{Relative wave amplitude}

\subsubsection{Dimensional analysis}

Using dimensional analysis, the amplitude of a transverse wave $A$ can be related to the geometrical and flow parameters. The functional relationship including all these parameters can be expressed as:

$$
\phi_{1}\left(A, D, B, L, l, h, p, U_{\lambda}, \mu, \rho, g, N, \gamma, n, f_{t w}, \mathrm{~d} D / \mathrm{d} x\right)=0
$$

where $p$ is the cylinder placement pattern (Zima \& Ackermann, 2002), $U_{\lambda}$ is average pore velocity with $U_{\lambda}=Q /(B D(1-\lambda))$ for emergent cylinders and $U_{\lambda}=Q /\left(B D\left(1-\lambda \frac{h}{D}\right)\right)$ for submerged cylinders, $\mu$ is dynamic viscosity, $\rho$ is water density, $N$ is the number of cylinders in a lateral row, and $\mathrm{d} D / \mathrm{d} x$ is flow depth gradient.

Given the link between $\gamma, n$, and $B$ (Eq. 1), that the geometrical parameters $B, l, L, h, N$ are constant in this case and that $p$ is fixed (in-line 
This is an Accepted Manuscript of an article published by Taylor \& Francis Group in Journal of Hydraulic Research on 22/07/2020, available online: https://www.tandfonline.com/doi/full/10.1080/00221686.2019.1647885.

arrangement), Eq. (4) can be expressed as a relation between seven dimensionless variables:

$$
A / D=\phi_{2}\left(D / h, \mathrm{~F}_{\mathrm{D}}, \mathrm{R}_{\mathrm{l}}, n, \mathrm{~S}_{\mathrm{l}}, \mathrm{d} D / \mathrm{d} x\right)
$$

where $\mathrm{F}_{\mathrm{D}}=U_{\lambda} / \sqrt{g D}$ is Froude number, $\mathrm{R}_{\mathrm{I}}=\rho U_{\lambda} l / \mu$ is cylinder Reynolds number, and $\mathbf{S}_{\mathrm{I}}=f_{t w} l / U_{\lambda}$ is Strouhal number assuming resonance conditions (vortex shedding frequency $=f_{t w}=f_{V}$, see section 4.1).

\subsubsection{Equations for emergent and submerged cylinders}

To the authors' knowledge, there is no equation for estimating relative wave amplitude, $A / D$, for square cylinder arrays. We therefore applied several equations derived for emergent or submerged circular cylinders.

For a staggered arrangement of emergent cylinders, and under resonance conditions, Zima and Ackermann (2002) proposed the following equation:

$$
\frac{A}{D}=A_{1} \mathrm{~S}_{\mathrm{I}}^{-2}
$$

where $A_{1}=K_{1} N(l / L)(l / B)^{2}$ and $K_{1}$ is a scale-independent constant (2.255).

For both staggered and in-line arrangements of emergent cylinders and under resonance conditions, Viero et al. (2016) (based on Defina and Pradella (2014)) model relative amplitude by the equation:

$$
\frac{A}{D}=\frac{A_{m}}{1+\frac{A_{D}}{N(l / L)} \frac{1}{\mathrm{~F}_{\mathrm{D}}}} \mathrm{F}_{\mathrm{D}}
$$

where $A_{m}$ and $A_{D}$ are two calibration parameters. When $\frac{A_{D}}{N(l / L)} \frac{1}{\mathrm{~F}_{\mathrm{D}}}$ is much larger than one, Eq. (7) can be simplified to:

$$
\frac{A}{D}=A_{2} \mathrm{~F}_{\mathrm{D}}^{2}
$$

where $A_{2}=K_{2} N(l / L)$, and $K_{2}=A_{m} / A_{D}$ is a function of $1 / n$.

For both staggered and in-line arrangements of emergent cylinders, the relative wave amplitude according to Jafari et al. (2010) is defined as :

$$
\frac{A}{D}=A_{3} \mathrm{~S}_{1}^{-0.53}
$$


This is an Accepted Manuscript of an article published by Taylor \& Francis Group in Journal of Hydraulic Research on 22/07/2020, available online: https://www.tandfonline.com/doi/full/10.1080/00221686.2019.1647885.

where $A_{3}=K_{3}(n / 2)^{0.26} N^{-1.16}\left(L_{x} / l\right)^{-0.7}\left(L_{y} / l\right)^{-0.44}, L_{x}$ and $L_{y}$ being the longitudinal and lateral spacings between the centers of two cylinders, $K_{3}=4.27$ and 1.47 for in-line and staggered arrangements of cylinders respectively.

Finally, according to Sarkar (2012), the relative wave amplitude for a random arrangement of submerged cylinders is given by

$$
\frac{A}{D}=\left[\frac{A_{4}}{A_{5} \frac{D}{h}-\pi}\right] \mathrm{S}_{\mathrm{I}}^{-2}
$$

where $A_{4}=K_{4}\left(\frac{l}{B}\right), A_{5}=2\left(\frac{L}{l}\right)^{2}$, and $K_{4}=6.62$.

\subsection{Square versus circular cylinders}

We recall here a number of important differences between square and circular cylinders. Firstly, the dimensionless drag force, also called drag force coefficient $C_{D}$, is higher for an isolated square cylinder than for an isolated circular cylinder: for $10^{4} \leq \mathrm{R}_{\mathrm{l}} \leq 10^{6}, C_{D}=2.1$ for a square cylinder, while $C_{D}=1.2$ for a circular cylinder (Hoerner, 1965). Secondly, the variation in $C_{D}$ with planar density $\lambda$ is converse for arrays of square and circular cylinders: $C_{D}$ increases with $\lambda$ for square cylinders and $0 \leq \lambda \leq 0.25$ (Buccolieri, Wigö, Sandberg, \& Di Sabatino, 2016; Leonardi \& Castro, 2010; Ludeña, Lopez, Rivière, \& Mignot, 2017, August), while $C_{D}$ decreases for circular cylinders with $\lambda$, for $0.0047 \leq \lambda \leq 0.12$ (Liu \& Zeng, 2017; H. Wang, Tang, Yuan, Lv, \& Zhao, 2014). Finally, the dimensionless frequencies of vortex shedding (Strouhal number $\mathrm{S}_{\mathrm{I}}$ ) behind square and circular cylinders are significantly different. For an isolated square cylinder, $\mathrm{S}_{\mathrm{I}}=0.10$ to 0.15 for $70 \leq R_{\mathrm{I}} \leq 2 \times 10^{4}$ (Okajima, 1982), while for an isolated circular cylinder, $S_{I}=0.20$ for $3 \times 10^{2} \leq R_{I} \leq 3 \times 10^{5}$ (Sumer \& Fredsoe, 2006).

\section{Results}

\subsection{Frequencies of seiche and vortex shedding}

The transverse seiche wave parameters at various $x / B$-positions are given in Table 2 for four cases (no measurements for cases AQ1W20 and AQ2W20). Frequencies $f_{D}$ and $f_{V}$ are the predominant frequencies measured from the flow depth fluctuation and transverse velocity fluctuation spectra respectively 
This is an Accepted Manuscript of an article published by Taylor \& Francis Group in Journal of Hydraulic Research on 22/07/2020, available online: https://www.tandfonline.com/doi/full/10.1080/00221686.2019.1647885.

(see section 4.5). These two frequencies characterize the transverse seiche wave. They are found to be either equal or very close to each other due to continuity in the transverse direction.

The vortex shedding frequency was estimated for flow case UQ1W40. Flow visualization was performed by sprinkling saw dust on the water surface (Fig. A, multimedia view). The vortex shedding frequency was $0.393 \mathrm{~Hz}$, which falls between $f_{D}$ and $f_{V}$. The lock-in process between surface waves and vortex shedding is clearly demonstrated. In addition, the video shows that the oscillating transverse motion across the full channel width is perfectly synchronized with the vortex shedding. In the remainder of this discussion, we therefore assume that frequency $f_{V}$ is a good approximation of vortex shedding frequency in these experiments and that the associated Strouhal number $\mathbf{S}_{\mathrm{I}}=f_{V} l / U_{\lambda}$.

Table 2: Seiching parameters at various longitudinal positions $x / B$ for two accelerated flow cases, AQ1W10 and AQ2W10, and uniform flow cases UQ1W40 and UQ2W47: $n$ is oscillation mode, $\sigma_{\max } / D$ is maximum relative standard deviation, $A / D$ is relative wave amplitude, $\mathrm{S}_{\mathrm{I}}=f_{V} l / U_{\lambda}$ is Strouhal number, $f_{V}$ and $f_{D}$ are measured frequencies based on transverse velocity and flow depth fluctuation spectra respectively, and $f_{t w}$ is the frequency of natural waves calculated using Eq. (3)

\begin{tabular}{|c|c|c|c|c|c|c|c|c|c|c|c|}
\hline Test cases & $x / B$ & $D / h$ & $n$ & $\sigma_{\max } / D(\%)$ & $A / D(\%)$ & $S_{1}$ & $\mathrm{R}_{\mathrm{l}}$ & $\mathrm{F}_{\mathrm{D}}$ & $f_{D}$ & $f_{V}$ & $f_{t w}$ \\
\hline \multirow[t]{5}{*}{ AQ1W10 } & 4.99 & 0.79 & 2 & 2.8 & 11.4 & - & 6003 & 0.14 & 0.634 & - & 0.596 \\
\hline & 8.57 & 0.77 & 2 & 3.2 & 13.1 & 0.41 & 6104 & 0.14 & 0.610 & 0.610 & 0.592 \\
\hline & 10.57 & 0.75 & 2 & 4.2 & 17.0 & - & 6281 & 0.15 & 0.610 & - & 0.584 \\
\hline & 12.14 & 0.71 & 2 & 4.9 & 21.5 & 0.37 & 6689 & 0.16 & 0.610 & 0.611 & 0.566 \\
\hline & 14.86 & 0.57 & 3 & 4.0 & 16.6 & - & 7903 & 0.21 & 0.848 & - & 0.776 \\
\hline \multirow[t]{5}{*}{ AQ2W10 } & 4.99 & 1.10 & 2 & 0.9 & 4.0 & 0.42 & 6853 & 0.13 & 0.708 & 0.707 & 0.696 \\
\hline & 8.57 & 1.08 & 2 & 1.2 & 5.1 & 0.40 & 6990 & 0.14 & 0.708 & 0.682 & 0.690 \\
\hline & 10.57 & 1.04 & 2 & 3.1 & 11.8 & 0.40 & 7306 & 0.15 & 0.708 & 0.707 & 0.681 \\
\hline & 12.14 & 0.98 & 2 & 4.7 & 18.8 & 0.37 & 7856 & 0.16 & 0.708 & 0.707 & 0.660 \\
\hline & 14.86 & 0.77 & 3 & 5.9 & 22.1 & 0.41 & 9502 & 0.22 & 0.950 & 0.955 & 0.888 \\
\hline UQ1W40 & 14.86 & 0.98 & 1 & 1.4 & 5.9 & 0.33 & 4844 & 0.10 & 0.398 & 0.385 & 0.335 \\
\hline UQ2W47 & 14.86 & 1.20 & 1 & 0.5 & 2.1 & 0.25 & 6173 & 0.12 & 0.384 & 0.384 & - \\
\hline
\end{tabular}


This is an Accepted Manuscript of an article published by Taylor \& Francis Group in Journal of Hydraulic Research on 22/07/2020, available online: https://www.tandfonline.com/doi/full/10.1080/00221686.2019.1647885.

\subsection{Seiche frequency for uniform vs. non-uniform flows}

The measured seiche frequencies were compared to the theoretical frequencies of natural waves in an open-channel, $f_{t w}$, calculated using Zhao's (Eq. 3), Dean's (combining Eq. 1 and Eq. 2) and Merian's equations. Figure 3 shows $f_{t w}$ as a function of $f_{D}$ at various $x / B$-positions for the four flow cases included in Table 2.

The results based on Dean's and Merian's equations are very similar because, in all four cases, these waves are effectively long waves in shallow water since $\tanh (n \pi D / B) \approx(n \pi D / B)(0.94 \leq \tanh (n \pi D / B) /(n \pi D / B) \leq 0.99)$.

While the seiche frequency is slightly overestimated by Dean's and Merian's equations, or slightly underestimated by Zhao's equation, which includes porosity, the three computed frequencies $f_{t w}$ remain a fairly good approximation to the seiche frequency and, in turn, to the vortex shedding frequency at resonance. This was observed for both uniform and non-uniform flows, irrespective of the $x / B$-position and submergence $D / h$-value.

These results indicate that the natural wave frequency equations for uniform flows appear to remain valid for non-uniform flows and for both emergent and slightly submerged cylinders. In non-uniform flows, frequency and mode are affected by a change in flow depth based on Eqs. (1) to (3) but there is no intrinsic effect of flow depth gradient (or velocity gradient) on seiche wave frequency. The results for accelerated cases AQ1W10 and AQ2W10 also show that two transverse standing waves with different frequencies can coexist at a very small longitudinal distance from each other. 
This is an Accepted Manuscript of an article published by Taylor \& Francis Group in Journal of Hydraulic Research on 22/07/2020, available online: https://www.tandfonline.com/doi/full/10.1080/00221686.2019.1647885.

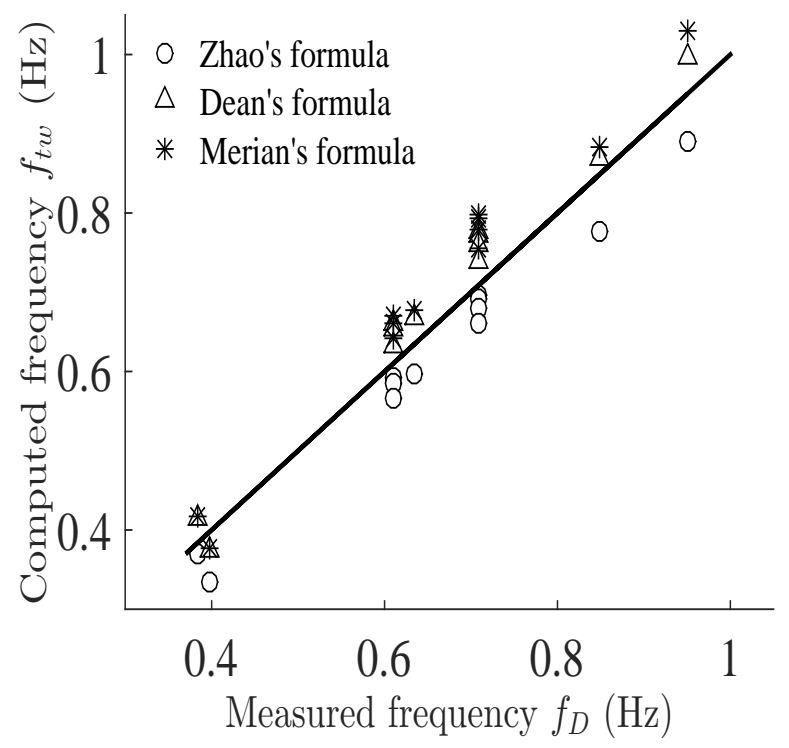

Figure 3: Measured seiche frequency $f_{D}$ at various $x / B$-positions with respect to computed frequency of naturel transverse waves, $f_{t w}$, using three formulae from the literature for the cases included in Table 2.

\subsection{Effect of wake interaction and seiche on Strouhal number}

For the four flow cases presented in Table $2, \mathrm{~S}_{\mathrm{I}}=0.25$ to 0.42 . The measured frequencies $f_{V}$ are thus two to three times larger than the values obtained for isolated square cylinders (section 3.3). Two physical processes can be responsible for this result: the lock-in process between vortex shedding and surface waves and the interaction between wakes within the cylinder arrays.

The effect of the lock-in process was evidenced in the previous section for the case of UQ1W40, as measured vortex shedding frequency, seiche frequencies $f_{V}$ and $f_{D}$, and natural waves frequency $f_{t w}$ fairly coincide. We can also assume that lock-in occurs for the other flow cases as $f_{t w}$ and $f_{V}$ are always close to each other. Regarding the effect of interacting wakes, we relied on the experimental work of Bokaian and Geoola (1984) on two interfering circular cylinders. The interaction is investigated in relation to the streamwise and transverse separation ratio $L / l$. For $L / l=2.2$ in both directions 
This is an Accepted Manuscript of an article published by Taylor \& Francis Group in Journal of Hydraulic Research on 22/07/2020, available online: https://www.tandfonline.com/doi/full/10.1080/00221686.2019.1647885.

(value in the present experiments), the Strouhal number of the downstream cylinder is not modified for $R_{\mathrm{l}}=5600$, and the lift and drag coefficients are very little modified for $2600 \leq R_{I} \leq 5900$. Based on this result, and on the fact that ratio $L / l$ is accounted for in Zhao's formula without significantly changing the waves frequency, we can thus conclude that seiche is primarily responsible for the increase in the Strouhal numbers.

\subsection{Parameters likely to impact seiche amplitude}

Based on Eq. (5), the likely dependence between relative amplitude $A / D$ and the six other dimensionless parameters was investigated. Only six flow cases were studied so we were not attempting to establish an equation suitable for both emergent and submerged square cylinders under uniform and nonuniform flows. On the other hand, we estimated the relative influence of each dimensionless parameter on the seiche amplitude in accordance with the flow conditions.

\subsubsection{Relative submergence}

The effect of $D / h$ was first quantified under uniform flow conditions (Fig. 4). For $D / h=0.98$, strong periodic free surface oscillations could be observed at lateral position $y / B=0.93$ (Fig. 4a). This position corresponds to an antinode (Fig. $4 \mathrm{~b}$ ), at which relative amplitude $A / D$ reaches $6 \%$ (Table 2), i.e. $A=3.4 \mathrm{~mm}$. Figure $4 \mathrm{~b}$ also shows that the oscillation mode $(n=1)$ and the anti-node and node locations were well-defined. For $D / h=1.20$, periodic oscillations were still clearly visible (Fig. 4a), with a $A / D$-value of $2.1 \%$ (Table 2), i.e. $A=1.5 \mathrm{~mm}$. This highlights the presence of seiche waves in the case of slight submergence as observed by Jafari et al. (2010) and Sarkar (2012) for submerged circular cylinders. On the other hand, the oscillation mode, nodes and anti-nodes are far less observable across the channel than in the emergent cylinder case (Fig. 4b). It should be noted that the oscillation mode can also be calculated $(n=1)$ using (i) the main oscillation frequency arising from the time series of flow depth and (ii) Eq. (3). The longitudinal profiles of $\sigma / D$ in Fig. 4c confirm that seiche magnitude is significantly smaller when cylinders are slightly submerged than when they are emergent. This may be ascribed to the damping effect of the high momentum flow above the cylinders on the oscillating lift force (due to vortex shedding) within the low momentum flow below the cylinders. 
This is an Accepted Manuscript of an article published by Taylor \& Francis Group in Journal of Hydraulic Research on 22/07/2020, available online: https://www.tandfonline.com/doi/full/10.1080/00221686.2019.1647885.

Figure $4 \mathrm{c}$ also indicates that, when cylinders are emergent (UQ1W40), noticeable changes in seiche magnitude can be observed along an anti-node line (at $y / B=0.93)$. Seiching decreases significantly, when approaching the inlet tank, as observed by Dupuis et al. (2016) for emergent circular cylinders. In addition, local maxima and minima can be identified along an anti-node line. For example, two local minima are separated by a distance of around $3.6 B$, suggesting the presence of a longitudinal wave with a wavelength significantly higher than the transverse wave (compare Fig. 4c to Fig. 4b). Unfortunately, no satisfactory explanation cannot be provided since $\sigma / D$ 's pattern is not the signature of a progressive longitudinal wave triggered by reflection phenomena (Zima \& Ackermann, 2002) or of the longitudinal component a metachronal wave (Viero et al., 2016).

The effect of $D / h$ was then quantified under non-uniform flow conditions. Transverse profiles of $\sigma / D$ at various $x / B$-positions are shown in Fig. 5 for the cases AQ1W10 and AQ2W10. In the latter case, longitudinal profiles of $\sigma / D$ and $D / h$ at $y / B=0.57$ (near an anti-node until $x / B=12.14$ ) are also illustrated in Fig. 6. The transition from cylinder submergence to emergence occurs between $x / B=10.57$ and 12.14. The results in both figures indicate that $A / D$ decreases with increasing $D / h$, when cylinders are submerged.

On the other hand, Fig. 5d suggests that the link between $A / D$ and $D / h$ is not unequivocal when cylinders are emergent. This is confirmed in Fig. 7a. The latter figure compares the $\sigma / D$ distribution in uniform flow case UQ1W40 with that in accelerated flow case AQ2W10 at a fixed relative submergence $(D / h=0.98)$ at two different downstream positions, $(x / B=14.86$ and 12.14$)$ respectively (table 2$)$. The relative amplitude $A / D$ is three times larger for the accelerated flow than for the uniform flow $(19 \%$ vs. $6 \%$ ), confirming that no role is played by $D / h$ for emergent cylinders.

Figure 6 also shows, for the AQ2W10 case, and when $D / h>1$, that the previously mentioned damping effect of the high momentum flow above the top of cylinders on the flow below the top of cylinders is not abrupt, but increases steadily with $D / h$.

The results for both uniform and non-uniform flows with $D / h>1$ are grouped together in Fig. 8a. The relative amplitude decreases rapidly from $12 \%$ to $2 \%$ for $D / h=1.04$ to 1.2 . Using Sarkar's equation to estimate $A / D$ is unsatisfactorily since these $A / D$ values are unrelated to the $S_{1}$-values. On the other hand, $A / D$ proportional to $a_{1} /\left(a_{2} D / h-1\right)$ with $a_{1}=0.4$ and $a_{2}=0.99$ is a good fit for the experimental data. However, the application of this equation to a flow case (Fig. 8b) appears to be unsuitable, when $D$ 
This is an Accepted Manuscript of an article published by Taylor \& Francis Group in Journal of Hydraulic Research on 22/07/2020, available online: https://www.tandfonline.com/doi/full/10.1080/00221686.2019.1647885.

tends towards $h$, and a linear fit would be more appropriate in this case. We can merely state that $A / D$ is strongly dependent on $D / h$ and is found to decrease with respect to $D / h$. As previously observed, when $D / h<1$, the relationship between $A / D$ and $D / h$ is not unequivocal for the Table 2 data (not shown here).

(a)
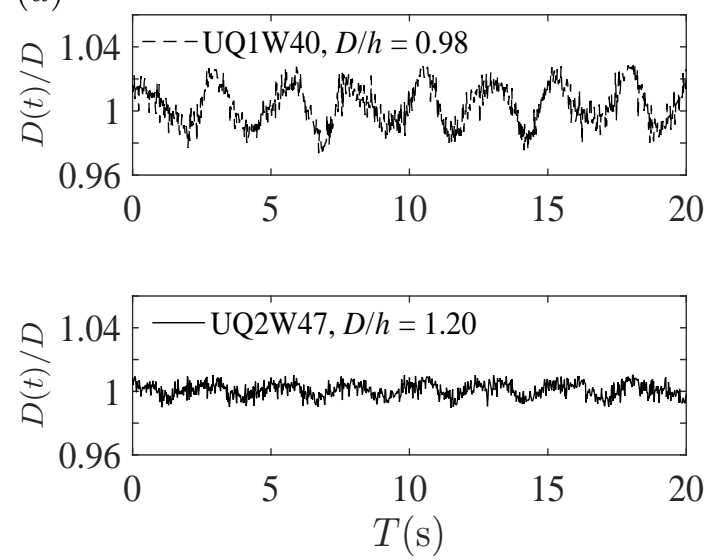

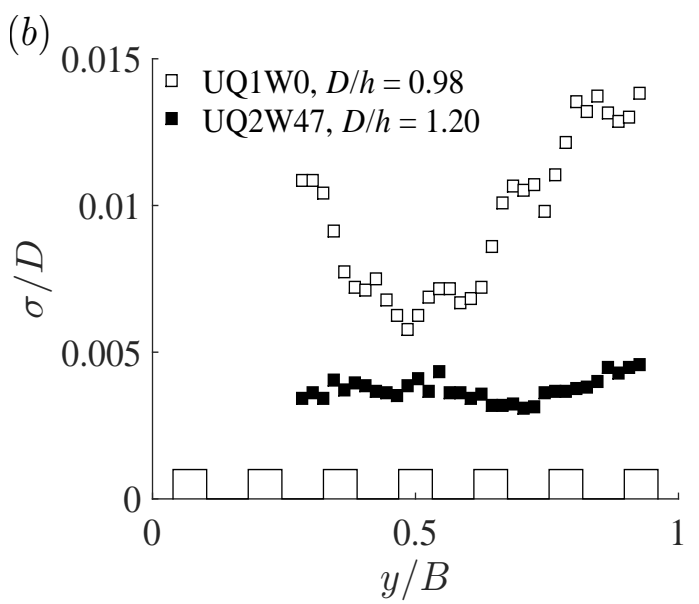

$(c)$

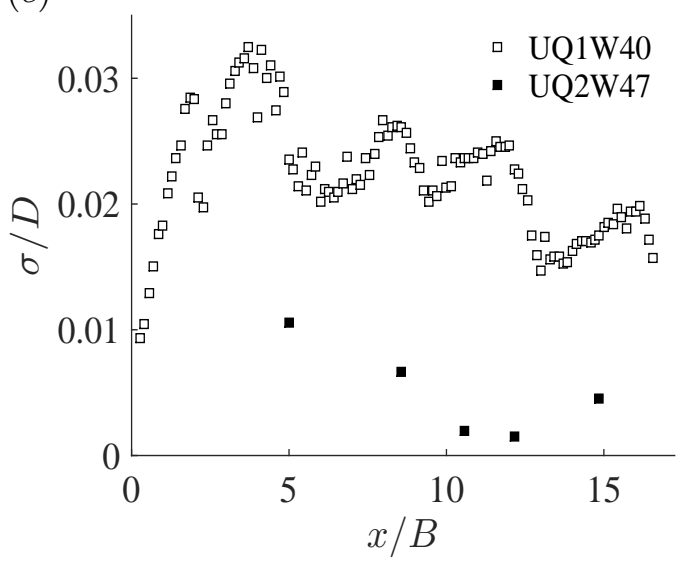

Figure 4: Uniform flows: (a) time series of instantaneous flow depth $D(t)$ with respect to time-averaged flow depth $D$, at lateral position $y / B=0.93$ and at downstream position $x / B=14.86$; (b) ratio of the standard deviation of flow depth fluctuation $\sigma$ to $D$ with respect to $y / B$, at $x / B=14.86$; (c) longitudinal variation in ratio $\sigma / D$ at $y / B=0.93$. 
This is an Accepted Manuscript of an article published by Taylor \& Francis Group in Journal of Hydraulic Research on 22/07/2020, available online: https://www.tandfonline.com/doi/full/10.1080/00221686.2019.1647885.

(a)

(c)
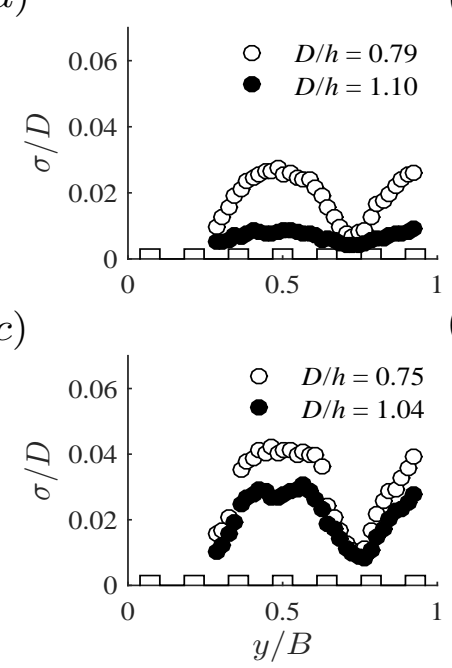

(b)

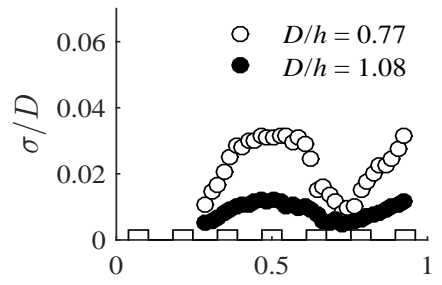

$(d)$

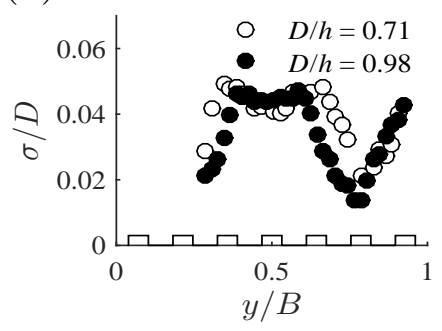

Figure 5: Ratio $\sigma / D$ for accelerated flow cases AQ1W10 (circles) and AQ2W10 (bullets) at downstream positions (a) $x / B=4.99$; (b) $x / B=8.57$; (c) $x / B=10.57$; (d) $x / B=12.14$.

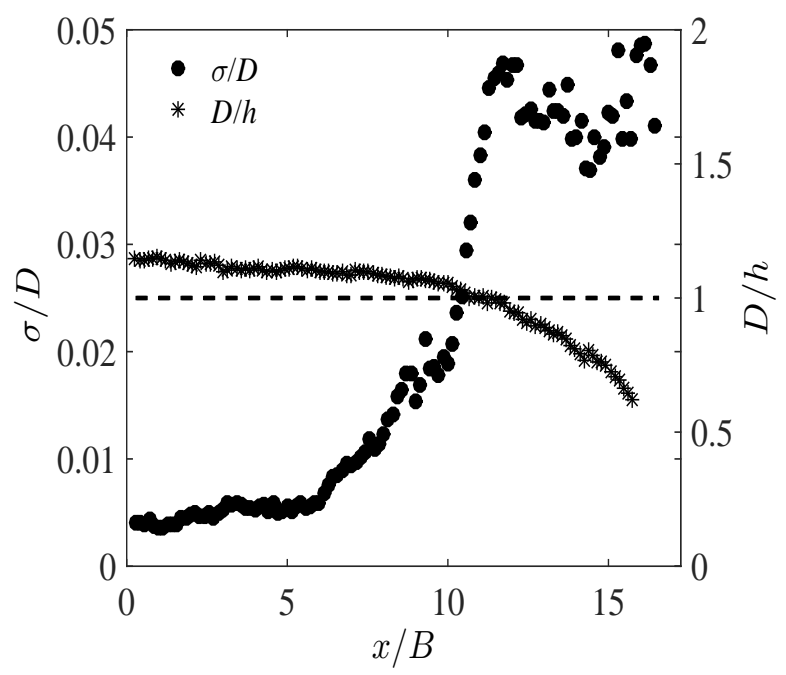

Figure 6: Ratio $\sigma / D$ and relative submergence $D / h$ with respect to downstream position $x / B$ at $y / B=0.57$ for case AQ2W10. 
This is an Accepted Manuscript of an article published by Taylor \& Francis Group in Journal of Hydraulic Research on 22/07/2020, available online: https://www.tandfonline.com/doi/full/10.1080/00221686.2019.1647885.

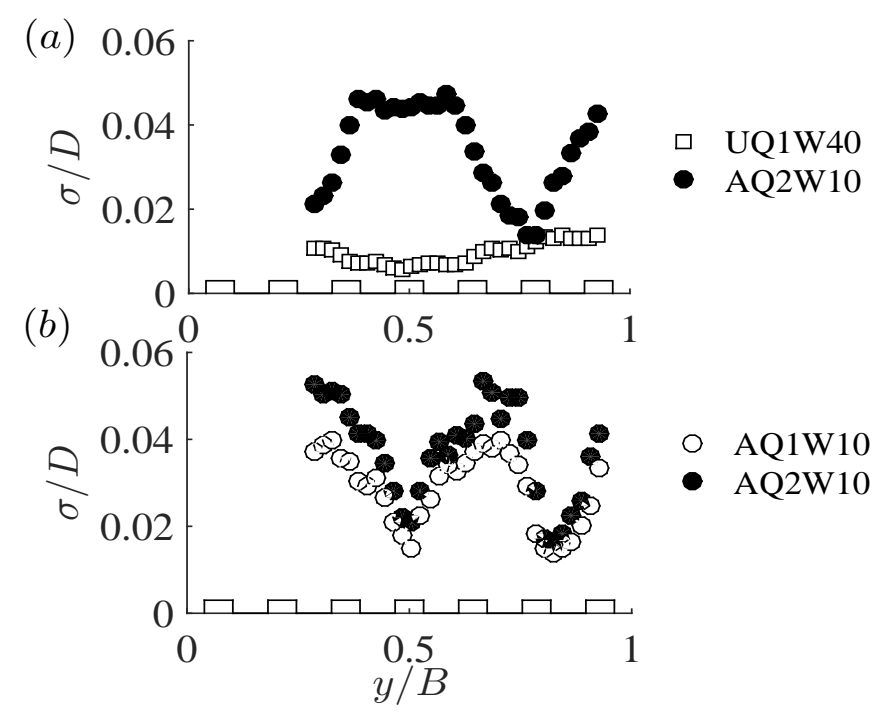

Figure 7: (a) Lateral distribution of ratio $\sigma / D$ at a fixed relative submergence, $D / h=0.98$, for cases UQ1W40 and AQ2W10; (b) Ratios $\sigma / D$ for cases AQ1W10 $(D / h=0.57)$ and AQ2W10 $(D / h=0.77)$ with respect to lateral position $y / B$ at downstream position $x / B=14.86$.

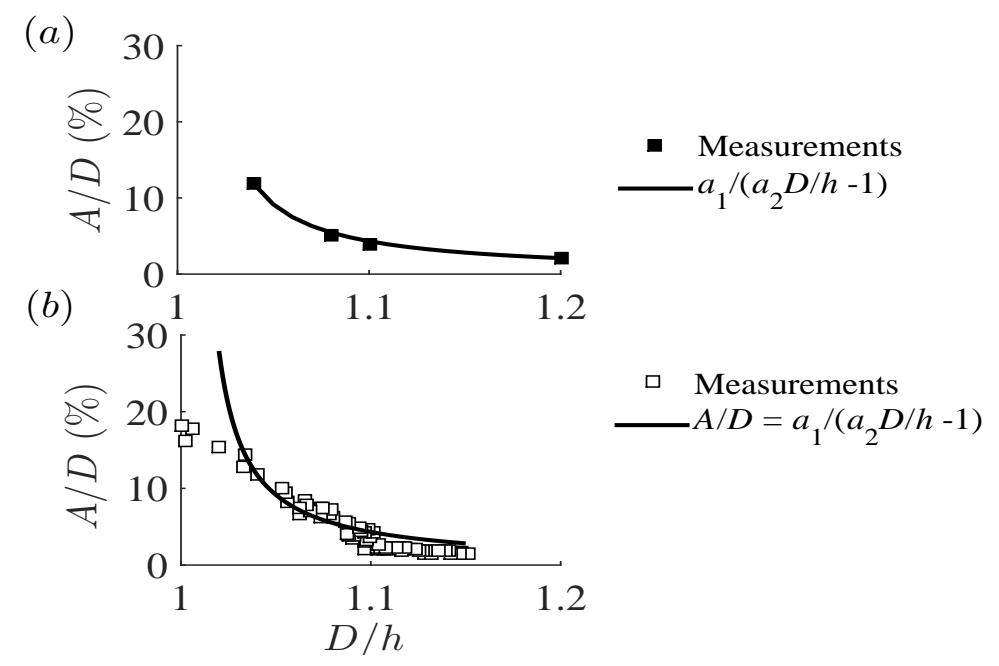

Figure 8: (a) Table 2 values for submerged cylinders and (b) measured and computed relative amplitude with respect to $D / h$ for case AQ2W10. 
This is an Accepted Manuscript of an article published by Taylor \& Francis Group in Journal of Hydraulic Research on 22/07/2020, available online:

https://www.tandfonline.com/doi/full/10.1080/00221686.2019.1647885.

\subsubsection{Froude number}

The next step was to assess the dependency of $A / D$ on the Froude number $F_{D}$, which was clearly demonstrated for emergent circular cylinders by Viero et al. (2016). Based on these authors' equation (Eq. (8)), the relative amplitude $A / D$ was plotted in Fig. 9a as a function of the square of Froude number, separating the data for emergent and submerged cylinders, and highlighting the $n$-number. For a fixed $\mathrm{F}_{\mathrm{D}}$, the decrease in seiche magnitude from emergence to submergence is visible for $n=2$. For a given $n$, the relative amplitude increases with respect to the Froude number under both emergent and submerged conditions. This could be a linear function of $\mathrm{F}_{\mathrm{D}}^{2}$ but additional measurements are required for confirmation. The linear link between $A / D$ and $\mathrm{F}_{\mathrm{D}}^{2}$ was tested for a longitudinal profile of $A / D$ in the AQ1W10 case (Fig. 9b). The experimental data were compared to the $A / D$-values computed from Eq. (8) and the coefficient $A_{2}$ was calibrated on the present data assuming that (a) $A_{2}$ is a constant independent of $n$ and (b) $A_{2}$ is proportional to $1 / n$ in keeping with Viero et al. (2016). Figure $9 \mathrm{~b}$ shows that a linear relationship between $A / D$ and $\mathrm{F}_{\mathrm{D}}^{2}$ is a good approximation. The results are slightly improved by considering $A / D$ proportional to $\frac{1}{n} \mathrm{~F}_{\mathrm{D}}^{2}$. The same results are obtained when plotting the $A / D$-data of Table 2 with respect to $\frac{1}{n} \mathrm{~F}_{\mathrm{D}}^{2}$.

The effect of Froude number on $A / D$ can be clearly seen in Fig. 10, which compares the longitudinal profile of $\sigma / D$ for UQ1W10 and AQ1W10; two cases with the same flow rate. The increase in Froude number for AQ1W10 in the second half of the flume is associated with a significant rise in seiche magnitude. 
This is an Accepted Manuscript of an article published by Taylor \& Francis Group in Journal of Hydraulic Research on 22/07/2020, available online: https://www.tandfonline.com/doi/full/10.1080/00221686.2019.1647885.

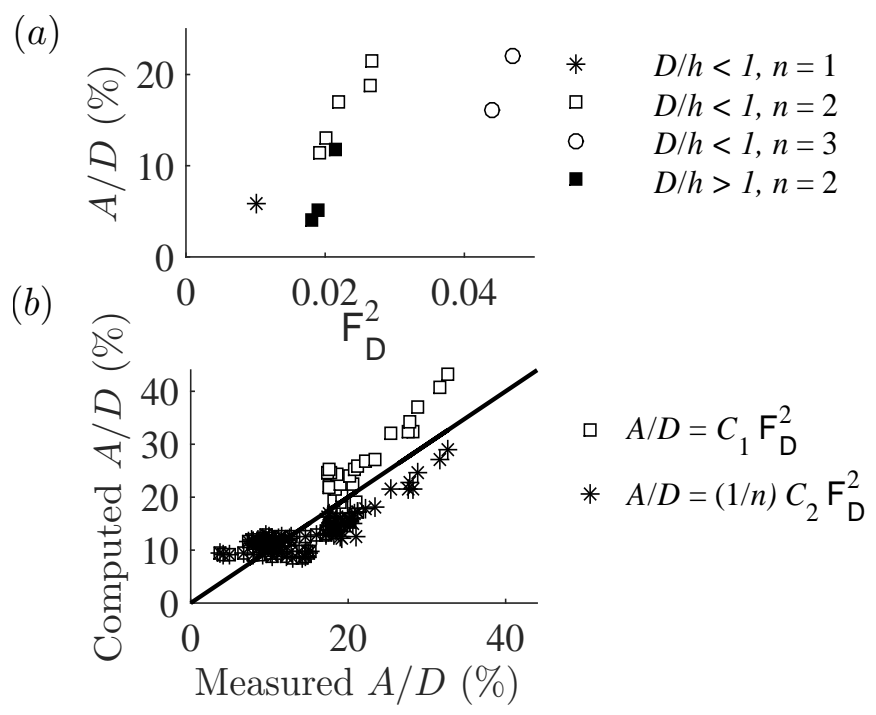

Figure 9: (a) Relative amplitude $A / D$ as a function of $\mathrm{F}_{\mathrm{D}}^{2}$ for Table 2 data; (b) measured $A / D$ vs computed $A / D$ using the Viero et al. (2016) equation with a calibrating coefficient $A_{2}=C_{1}$ and $A_{2}=(1 / n) C_{2}$, flow case AQ1W10.

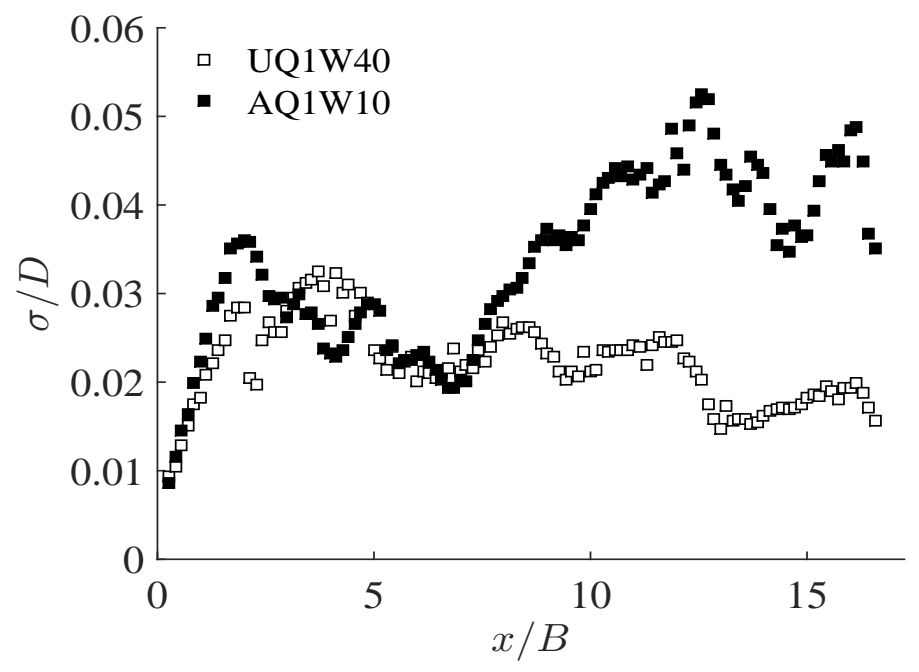

Figure 10: Uniform flow vs. accelerated flow: longitudinal variation in $\sigma / D$ at $y / B=0.93$ for cases UQ1W40 and AQ1W10. 
This is an Accepted Manuscript of an article published by Taylor \& Francis Group in Journal of Hydraulic Research on 22/07/2020, available online:

https://www.tandfonline.com/doi/full/10.1080/00221686.2019.1647885.

\subsubsection{Strouhal and Reynolds numbers}

The Zima and Ackermann (2002) equation, Eq. (6), was first tested using the study data. The relative amplitude $A / D$ is plotted in Fig. 11a with respect to $\mathrm{S}_{\boldsymbol{I}}^{-2}$ for the Table 2 data. The results are comparable to those obtained for the Froude number in Fig. 9a. This can be explained by the relationship between $S_{I}$ and $F_{D}$ at resonance. If vortex shedding frequency is approximated using Merian's equation, then $\mathrm{S}_{\mathrm{I}}=l n /\left(2 B \mathrm{~F}_{\mathrm{D}}\right)$. For a given $n$-value, $A / D$ proportional to $\mathrm{F}_{\mathrm{D}}^{2}$ is equivalent to $A / D$ proportional to $\mathrm{S}_{1}^{-2}$. However, Fig. 11a shows that coefficient $A_{1}$ in Eq. (6) is in practice dependent on the oscillation mode and is not a constant

As a second step, we tested the Jafari et al. (2010) equation, which relates $A / D$ to both $\mathrm{S}_{\mathrm{I}}$ and $n$ (Fig. 11b). Focusing on emergence conditions, the data with $n=1,2$, and 3 are more aligned than in the previous case, thereby confirming the dual influence of $\mathrm{S}_{\mathrm{I}}$ and $n$.

Finally, we tested the likely dependence of relative amplitude on cylinder Reynolds number $\mathrm{R}_{\mathrm{l}}$ (not shown here). For a given $n, A / D$ increases with $\mathrm{R}_{\mathrm{l}}$, i.e. with $U_{\lambda}$. This effect was already highlighted by the relationship between $A / D$ and Froude number.

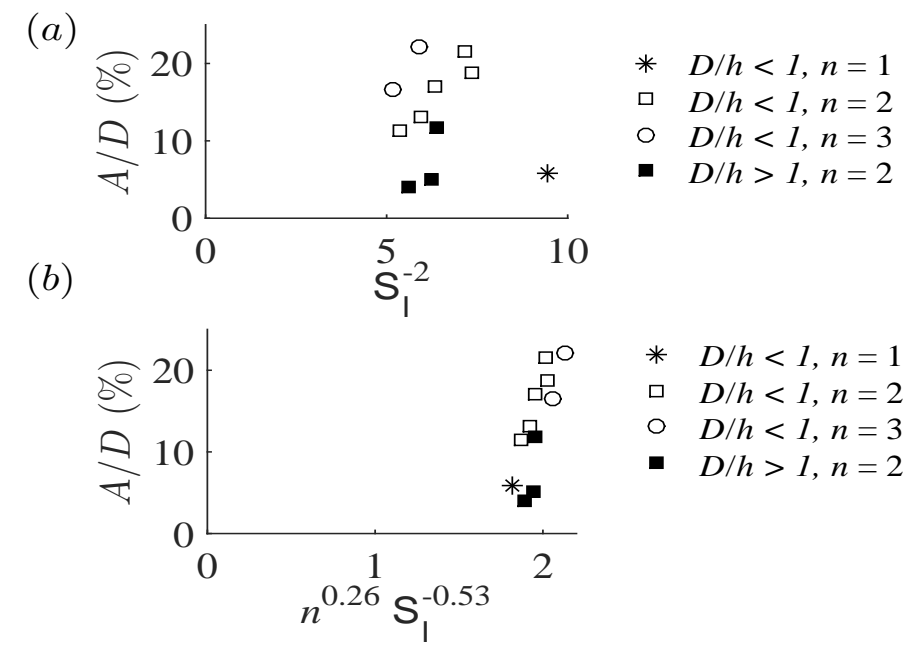

Figure 11: (a) Relative submergence $A / D$ with respect to $\mathrm{S}_{1}^{-2}$ based on the Zima and Ackermann (2002) equation, see Eq. (6); (b) $A / D$ with respect to $n^{0.26} \mathrm{~S}_{\boldsymbol{I}}^{-0.53}$ based on Jafari et al. (2010), see Eq. (9). 
This is an Accepted Manuscript of an article published by Taylor \& Francis Group in Journal of Hydraulic Research on 22/07/2020, available online:

https://www.tandfonline.com/doi/full/10.1080/00221686.2019.1647885.

\subsubsection{Oscillation mode}

Seiche frequency and wavelength are both dependent on the oscillation number $n$ (Eqs. (1) to (3)), so we could expect that relative amplitude is also be related to $n$. This is supported by both Fig. 9a or Fig. 11a. Relative amplitude is strongly dependent on couple $\left(\mathrm{F}_{\mathrm{D}}, n\right)$ or couple $\left(\mathrm{S}_{\mathrm{l}}, n\right)$, confirming the results of Viero et al. (2016), and Jafari et al. (2010) respectively.

When the flow is non-uniform, the longitudinal change in mode oscillation from $n=2$ to $n=3$ for both flows AQ1W10 and AQ2W10 (Table 2, Figs. 5a and $7 \mathrm{~b}$ ) also affects the relative standard deviation $\sigma / D$ at a fixed $y$-value.

\subsubsection{Flow depth gradient}

It was not possible to demonstrate that flow depth gradient can intrinsically alter the relative amplitude. This likely effect is concealed by the dependence of $A / D$ on the Froude number, which varies significantly in the longitudinal direction in non-uniform flows, as shown in Fig. 9b for the AQ1W10 case.

\subsection{Influence of seiche wave on streamwise mean flow and turbulence statistics}

Preliminary velocity measurements were taken to estimate the effect of a seiche wave on streamwise mean flow and turbulence statistics for two accelerated cases. Velocities were measured between $y / B=0.29$ and 0.57 .

Figure 12a shows transverse distributions of the depth-averaged streamwise velocity $U_{D}$, normalized by the bulk velocity $U_{Q}$, which were measured at $x / B=8.57$ and $x / B=12.14$ for the two flow cases. We focus on the three fast stream regions at $y / B=0.29,0.43$ and 0.57 . When the cylinders are slightly submerged (case AQ2W10 with $D / h=1.08$ ), the local peak velocities are equal at these three $y / B$-values. Figure $5 \mathrm{~b}$ shows that $y / B=0.29$ is a node, while the positions $y / B=0.43$ and 0.57 are close to an anti-node. The water surface oscillations do not seem therefore to have an impact on the streamwise mean flow, when $D / h>1$.

On the other hand, when the cylinders are emergent $(D / h<1)$, the local peak velocity observed at $y / B=0.29$ is always lower than the local peaks at $y / B=0.43$ and 0.57 . For the case AQ1W10 with $D / h=0.77$ and $D / h=0.71$ and the case AQ2W10 with $D / h=0.98$, position $y / B=0.29$ is a node, while positions $y / B=0.43$ and 0.57 are close to an anti-node (Figs. 
This is an Accepted Manuscript of an article published by Taylor \& Francis Group in Journal of Hydraulic Research on 22/07/2020, available online: https://www.tandfonline.com/doi/full/10.1080/00221686.2019.1647885.

$5 \mathrm{~b}$ and $5 \mathrm{~d})$. The velocity increase from a node to an anti-node is significant (30\% for AQ1W10 with $D / h=0.77$ and AQ2W10 with $D / h=0.98$, and $44 \%$ for AQ1W10 with $D / h=0.71)$. Figure $12 \mathrm{~b}$ also shows that water surface oscillations can alter the vertical distribution of mean velocity over the whole depth.

It should be added that the velocity deficit observed at $y / B=0.29$ (node) cannot be ascribed to lateral boundary layer influence. According to Nezu and Rodi (1985, August), as $B / D>5$ for both accelerated flows, the sidewalls effects are negligible for $0.11<y / B<0.89$.

The effects of seiching on the standard deviation of transverse and streamwise velocity fluctuations are shown in Figs. 13a and 13b respectively. Transverse velocity fluctuations are higher near the node region than near the anti-node region (24 to 40\%) for the four flow conditions studied, which is consistent with the definition of nodes and anti-nodes. No similar result can be observed for the streamwise velocity fluctuations, highlighting that the seiche waves studied here are predominately associated with an oscillating transverse motion of water. Figure 13a suggests that, when cylinders are slightly submerged, transverse velocity fluctuations might be too small compared to the mean bulk flow to alter the streamwise momentum of the mean flow as previously shown in Fig. 12a. All these results suggest that seiche waves can be responsible for discrepancies, from one elementary cylinder pattern to another, in (a) the streamwise mean velocity, when cylinders are emergent; and (b) transverse velocity fluctuation, when cylinders are emergent and submerged. 
This is an Accepted Manuscript of an article published by Taylor \& Francis Group in Journal of Hydraulic Research on 22/07/2020, available online: https://www.tandfonline.com/doi/full/10.1080/00221686.2019.1647885.
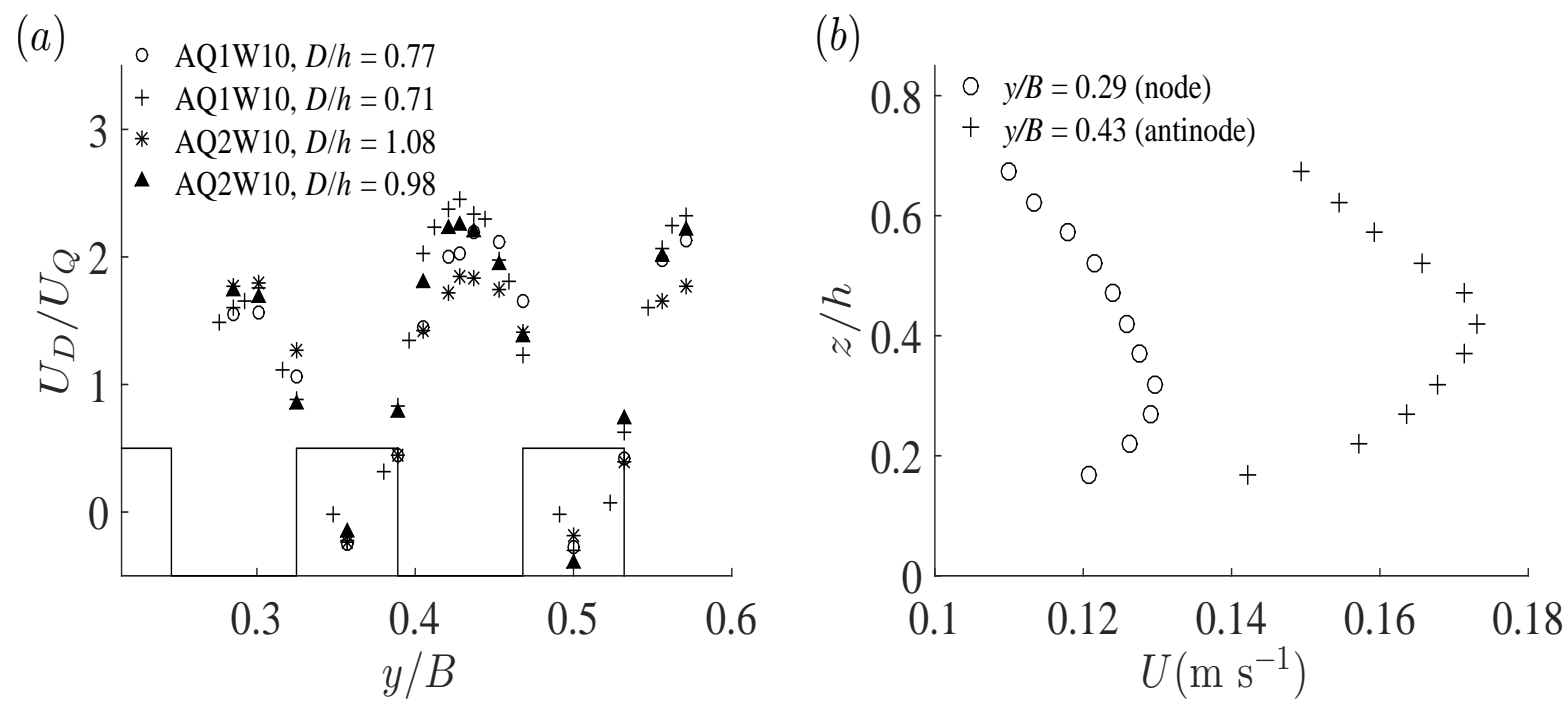

Figure 12: (a) Lateral distributions of depth-averaged streamwise velocity $U_{D}$, normalized by bulk velocity $U_{Q}=Q /(B D)$, for two accelerated flows at $x / B=8.57$ and 12.14 ; (b) vertical distribution of local streamwise mean velocity $U$ for case AQ1W10 at $x / B=8.57$ where $D / h=0.77$.
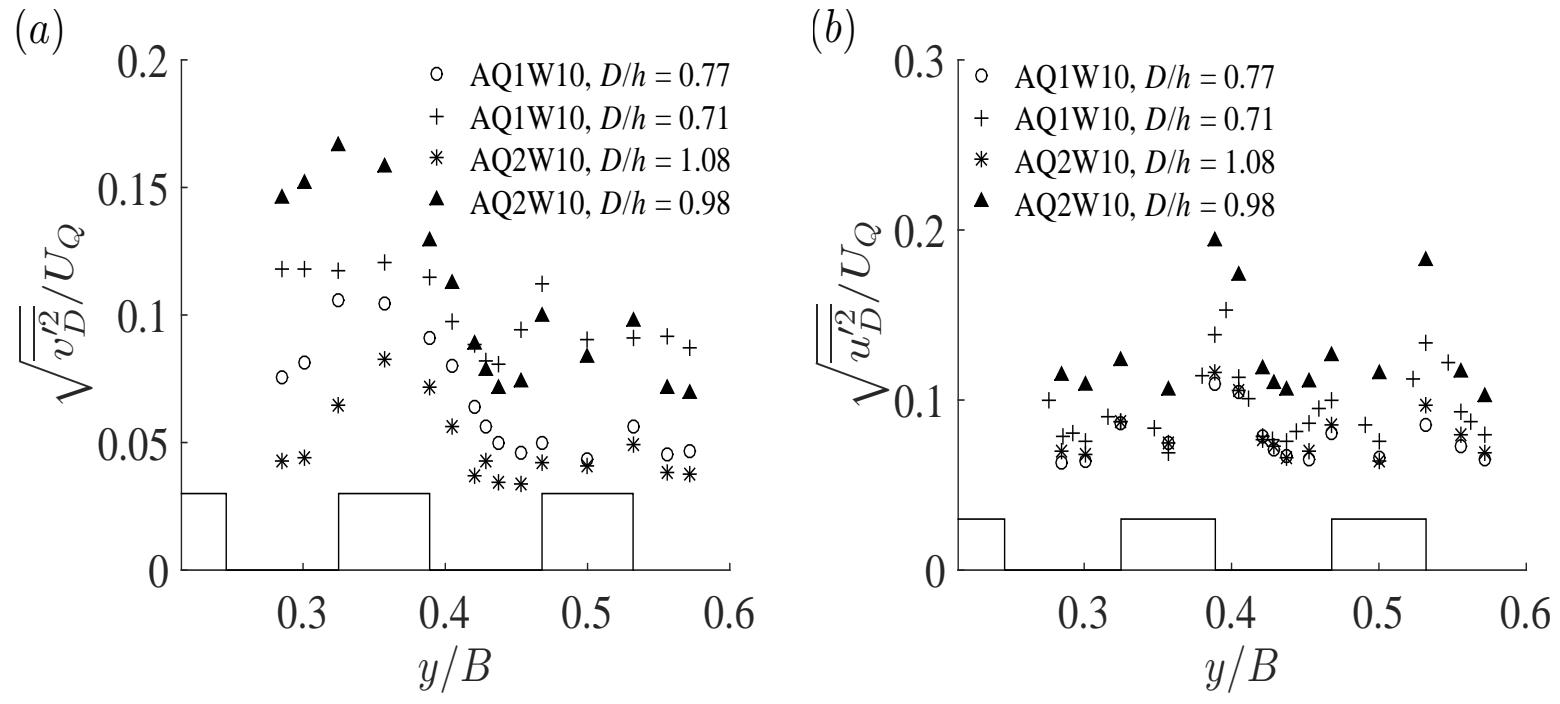

Figure 13: Depth-averaged standard deviations of (a) transverse and (b) streamwise velocity fluctuations with repect to lateral distance $y / B$. Normalisation by bulk velocity. 
This is an Accepted Manuscript of an article published by Taylor \& Francis Group in Journal of Hydraulic Research on 22/07/2020, available online:

https://www.tandfonline.com/doi/full/10.1080/00221686.2019.1647885.

\section{Conclusions}

Steady uniform and non-uniform flows through an array of emerged and slightly submerged square cylinders are investigated in a laboratory openchannel flume. We have focused on the transverse seiche waves induced by vortex shedding behind the cylinders. Seiche magnitude is quantified by the relative wave amplitude $A / D$. The six flow cases studied feature a relative submergence $D / h$ of between 0.47 to 1.20 and a relative amplitude $A / D$ of between $2 \%$ and $38 \%$. The main findings of this study are:

- Periodic water surface oscillations were observed for both uniform and non-uniform flows, and both emergent and slightly submerged cylinders. A lock-in process between vortex-shedding and natural transverse waves occurred at each longitudinal position. When the flow was nonuniform, this resulted in the co-existence of two transverse standing waves with different modes at a small distance from each other.

- The measured seiche frequencies (arising from spectra of flow depth fluctuation or transverse velocity fluctuation) could be approximated by applying natural wave frequency equations developed for long waves in shallow water. These equations were found to be valid for both uniform and non-uniform flows and for both emergent and slightly submerged cylinders.

- The observed Strouhal numbers (dimensionless frequencies of vortex shedding) were two or three times higher than the Strouhal number for an isolated square cylinder due to the lock-in process.

- The relative wave amplitude $A / D$ was significantly lowered, when initially emergent cylinders became slightly submerged. This was observed, in particular, for a fixed Froude number and a fixed oscillation mode value.

- When cylinders were submerged, $A / D$ was found to be essentially influenced by the relative submergence $D / h$. It decreased from $12 \%$ to $2 \%$ for $D / h=1.04$ to 1.20 .

- When cylinders were emergent, $A / D$ was found to be mainly influenced by the Froude number and the oscillation mode. Owing to the resonance phenomenon (lock-in process), this was equivalent to control by the Strouhal number and the mode $n$. 
This is an Accepted Manuscript of an article published by Taylor \& Francis Group in Journal of Hydraulic Research on 22/07/2020, available online: https://www.tandfonline.com/doi/full/10.1080/00221686.2019.1647885.

- Finally, the flow structure was found to be altered by the seiche waves. For emergent cylinders, the streamwise mean velocity was found to be lower at nodes than at anti-nodes (up to 44\%). For both emergent and submerged cylinders, the transverse velocity fluctuations were higher at nodes than at anti-nodes (up to $40 \%$ ).

\section{Acknowledgments}

The authors wish to thank André Paquier and Marc Chatelain for their invaluable advice.

\section{Funding}

Meriem Chetibi conducted her experiments at Irstea within the framework of a research project funded by the French National Research Agency (Flowres project, Grant No. ANR-14-CE03-0010, https://flowres.irstea.fr/en/).

\section{Notation}

The following symbols are used in this paper:

$A=$ wave amplitude at a given $x$-position $(\mathrm{mm})$

$B=$ channel width $(\mathrm{mm})$

$D=$ time-averaged water depth $(\mathrm{mm})$

$D(t)=$ instantaneous water depth $(\mathrm{mm})$

$D / h=$ relative submergence $(-)$

$\mathrm{F}_{\mathrm{D}}=$ Froude number based on water depth $D$ and velocity $U_{\lambda}(-)$

$f_{D}=$ predominant frequency of the transverse seiche wave based on measured water depth fluctuation spectrum $(\mathrm{Hz})$

$f_{t w}=$ theoretical frequency of the natural transverse waves, see Eq. (3) $(\mathrm{Hz})$

$f_{V}=$ predominant frequency of the transverse seiche wave based on the measured transverse velocity fluctuation spectrum $(\mathrm{Hz})$

$h=$ height of a square cylinder $(\mathrm{mm})$

$l=$ width of a square cylinder $(\mathrm{mm})$

$L=$ distance between the centers of two adjacent square cylinders (mm)

$N=$ number of square cylinders in a transverse row $(-)$

$n=$ oscillation mode $(-)$ 
This is an Accepted Manuscript of an article published by Taylor \& Francis Group in Journal of Hydraulic Research on 22/07/2020, available online:

https://www.tandfonline.com/doi/full/10.1080/00221686.2019.1647885.

$Q=\operatorname{discharge}\left(\mathrm{L} \mathrm{s}^{-1}\right)$

$\mathrm{R}_{\mathrm{I}}=$ Reynolds number based on width $l$ and velocity $U_{\lambda}(-)$

$S_{0}=$ The longitudinal bed slope of the channel $(-)$

$\mathrm{S}_{\mathrm{I}}=$ Strouhal number based on width $l$ and velocity $U_{\lambda}(-)$

$U_{\lambda}=$ average pore velocity $\left(\mathrm{m} \mathrm{s}^{-1}\right)$

$W=$ downstream weir height $(\mathrm{mm})$

$\gamma=$ transverse wavelength $(\mathrm{mm})$

$\lambda=$ planar density of square cylinders $(-)$

$\lambda_{\mathrm{f}}=$ frontal density of square cylinders $(-)$

$\sigma=$ standard deviation of water depth fluctuations $(\mathrm{mm})$

$\sigma_{\max }=$ maximum value of $\sigma$ at a given $x$-position (mm)

\section{References}

Bokaian, A., \& Geoola, F. (1984). Wake-induced galloping of two interfering circular cylinders. Journal of Fluid Mechanics, 146, 383-415.

Buccolieri, R., Wigö, H., Sandberg, M., \& Di Sabatino, S. (2016). Direct measurements of the drag force over aligned arrays of cubes exposed to boundary-layer flows. Environmental Fluid Mechanics, 17(2), 373-394.

Chagot, 1., Moulin, F., \& Eiff, O. (2017, June). Combined effects of relative submergence and roughness aspect ratio on canopy flows. Paper presented at the 4th International Symposium of Shallow Flows on Eindhoven University of Technology.

Chen, J.-T., Lee, J.-W., Wu, C.-F., \& Lee, Y.-T. (2013). Study on harbor resonance and focusing by using the null-field biem. Engineering Analysis with Boundary Elements, 37(7-8), 1107-1116.

Dean, R., \& Dalrymple, R. (1991). Water wave mechanics for engineers and scientists. World scientific publishing co. pte. ltd.

Defina, A., \& Pradella, I. (2014). Vortex-induced cross-flow seiching in cylinder arrays. Advances in Water Resources, 71, 140-148.

Dupuis, V., Proust, S., Berni, C., \& Paquier, A. (2016). Combined effects of bed friction and emergent cylinder drag in open channel flow. Environmental Fluid Mechanics, 16(6), 1173-1193.

Florens, E., Eiff, O., \& Moulin, F. (2013). Defining the roughness sublayer and its turbulence statistics. Experiments in Fluids, 54(4), 1-15.

Ghomeshi, M., Mortazavi-Dortazavi, S.-A., \& Falconer, R. (2007). Amplitude of wave formation by vortex shedding in open channels velocimeter 
This is an Accepted Manuscript of an article published by Taylor \& Francis Group in Journal of Hydraulic Research on 22/07/2020, available online: https://www.tandfonline.com/doi/full/10.1080/00221686.2019.1647885.

data. Journal of Applied sciences, 7(24), 3927-3934.

Goring, D.-G., \& Nikora, V.-I. (2002). Despiking acoustic doppler velocimeter data. Journal of Hydraulic Engineering-Asce, 128(1), 117-126.

Hoerner, S. (1965). Fluid dynamic drag. McGraw Hill Higher Education.

Ilich, K. (2006). Origin of continental shelf seiches (Unpublished doctoral dissertation). Environmental Systems Engineering, Fremantle, Western Australia.

Jafari, A., Ghomeshi, M., Bina, M., \& Kashefipour, S.-M. (2010). Experimental study on ten modes of transverse waves due to vertical cylinders in open channels. Journal of Food Agriculture and Environement, 8(2), 949-955.

Leonardi, S., \& Castro, I. P. (2010). Channel flow over large cube roughness: a direct numerical simulation study. Journal of Fluid Mechanics, 651, 519-539.

Liu, X., \& Zeng, Y. (2017). Drag coefficient for rigid vegetation in subcritical open-channel flow. Environmental Fluid Mechanics, 17(5), 1035-1050.

Ludeña, S.-G., Lopez, D., Rivière, N., \& Mignot, E. (2017, August). Extreme flood flow in an increasingly urbanized floodplain: an experimental approach. In Proceedings of the 37th IAHR world congress. Kuala Lumpur, Malaysia.

Nezu, I., \& Rodi, W. (1985, August). Experimental study on secondary currents in open channel flow. In Proceedings of the 21st IAHR congress (p. 115-119). Melbourne.

Okajima, A. (1982). Strouhal numbers of rectangular cylinders. Journal of Fluid Mechanics, 123, 379-398.

Proust, S., Berni, C., Boudou, M., Chiaverini, A., Dupuis, V., Faure, J.-B., ... Eiff, O. (2016, October). Predicting the flow in the floodplains with evolving land occupations during extreme flood events (FlowRes ANR project). In Proceedings of the 3rd European Conference on Flood Risk Management. Lyon-France.

Rabinovich, A. B. (2009). Seiches and harbor oscillations. In Handbook of coastal and ocean engineering (p. 193-236).

Rouzes, M., Moulin, F. Y., Florens, E., \& Eiff, O. (2018). Low relativesubmergence effects in a rough-bed open-channel flow. Journal of Hydraulic Research, 1-28.

Sarkar, A. (2012). Vortex-excited transverse surface waves in an array of randomly placed circular cylinders. Journal of Hydraulic Engineering, 138(7), 610-618. 
This is an Accepted Manuscript of an article published by Taylor \& Francis Group in Journal of Hydraulic Research on 22/07/2020, available online: https://www.tandfonline.com/doi/full/10.1080/00221686.2019.1647885.

Sumer, M. B., \& Fredsoe, J. (2006). Hydrodynamics around cylindrical structures. World scientific publishing co. pte. ltd.

Viero, D. P., Pradella, I., \& Defina, A. (2016). Free surface waves induced by vortex shedding in cylinder arrays. Journal of Hydraulic Research, 55(1), 16-26.

Wang, G., Zheng, J., Liang, Q., \& Zheng, Y. (2014). Analytical solutions for oscillations in a harbor with a hyperbolic-cosine squared bottom. Ocean Engineering, 83, 16-23.

Wang, H., Tang, H., Yuan, S., Lv, S., \& Zhao, X. (2014). An experimental study of the incipient bed shear stress partition in mobile bed channels filled with emergent rigid vegetation. Science China Technological Sciences, 57(6), 1165-1174.

Zhao, K., Cheng, N.-S., \& Huang, Z. (2014). Experimental study of freesurface fluctuations in open-channel flow in the presence of periodic cylinder arrays. Journal of Hydraulic Research, 52(4), 465-475.

Zima, L., \& Ackermann, N. L. (2002). Wave generation in open channels by vortex shedding from channel obstructions. Journal of Hydraulic Engineering, 128(6), 596-603. 